Klassisch, atypisch, latent, refraktär

\title{
Die Zöliakie hat viele Gesichter
}

\author{
Die Pathogenese der Zöliakie ist multifaktoriell. Sie umfasst \\ neben der Glutenunverträglichkeit auch genetische und immuno- \\ logische Faktoren. Der Verlauf der Erkrankung ist individuell \\ sehr unterschiedlich. Die einzige bisher verfügbare Therapie \\ ist die glutenfreie Diät.
}

Hoffentlich glutenfrei - Diätfehler trüben die Prognose bei Zöliakie.
_ „Die Zöliakie sollte bei allen Patienten mit unklaren gastrointestinalen $\mathrm{Be}$ schwerden einschließlich Fatigue und Anämie diskutiert werden“, sagte Prof. Elke Roeb von der Medizinischen Universitätsklinik in Gießen. Die Prävalenz der Erkrankung liegt in Deutschland bei $0,5-1 \%$. Sie kann sich in jedem Lebensalter manifestieren.

\section{Gluten, Gene und Immunsystem}

Die Pathogenese der Zöliakie umfasst Umwelt- bzw. Ernährungsfaktoren, d.h. Gluten, aber auch genetische und immunologische Faktoren. So besteht eine strenge Abhängigkeit von den HLAHaptotypen DQ2 und DQ8. Auch konnte in Zwillingsstudien die starke genetische Suszeptibilität nachgewiesen werden. Gluten selbst enthält mehr als 100 Proteine. „Ursächlich handelt es sich bei der Zöliakie um eine genetisch determinierte Unverträglichkeit von Gliadin, einem Protein im Gluten“, so Roeb. Dies führt bei Zöliakiepatienten zu einer immunologischen Reaktion mit Beteiligung der Lymphozyten. Folge ist ein Umbau der Dünndarmmukosa mit Zottenatrophie und Kryptenhyperplasie. Daraus resultiert klassischerweise eine Malabsorption.

\section{Unterschiedliches klinisches Bild}

Das klinische Bild der Zöliakie kann stark variieren. Das Spektrum der klinischen Manifestationen reicht von weitgehend asymptomatischen Verläufe bis hin zu vielfältigen intestinalen Symptomen wie Diarrhö, Steatorrhö und Maldigestion mit konsekutivem Eisenmangel bzw. Osteoporose durch Vitamin-D-Mangel. Dies führt zum Ge- wichtsverlust bzw. zu einem Wachstumsstillstand bei Kindern. Daneben gib es atypische Manifestationen in Form von neurologisch-psychiatrischen Symptomen wie zerebellärer Ataxie, Autismus oder Depression bzw. gynäkologischen Störungen wie Amenorrhoe bzw. Aborte. An der Haut kann sich die Zöliakie als Dermatitis herpetiformis Duhring manifestieren und auch eine isolierte Leberwerterhöhung kann Ausdruck dieser Erkrankung sein.

\section{Serologie als Screening}

Der wichtigste Baustein bei der Zöliakiediagnostik ist die Bestimmung der Transglutaminase-IgA-Antikörper zusammen mit Gesamt-IgA. Bei über $90 \%$ der Erkrankten sind diese Antikörper deutlich positiv, und bei fehlenden Antikörpern ist die Erkrankung mit 90\%iger Sicherheit ausgeschlossen. Bei grenzwertigen Antikörpertitern oder bei einem IgA-Mangel empfiehlt sich die Bestimmung der IgG-Antikörper gegen deamidierte Gliadine.

Die Frage, ob bei einer starken Erhöhung der Transglutaminase-IgA-Antikörper noch eine zusätzlich histologische Sicherung durch Duodenalbiopsien angestrebt werden muss, wird kontrovers diskutiert. Für die routinemäßige Endoskopie spricht, dass damit andere Differenzialdiagnosen wie der $\mathrm{M}$. Whipple oder eine eosinophile Enteritis ausgeschlossen werden können. Da sich die für die Zöliakie typischen Schleimhautveränderungen mosaikmäßig verteilen, sollten mindestens vier Biopsien aus der Pars descendens duodeni entnommen werden. „Wichtig ist es, dass die Diagnose frühzeitig gestellt wird, bevor gravierende Lang- zeitfolgen wie Osteoporose, Infertilität oder Dünndarmlymphome aufgetreten sind“, betonte Roeb.

\section{Bewährte und künftige Behandlungsstrategien}

Die einzige sinnvolle Therapie ist eine strikt glutenfreie Diät. Diese ist nicht nur im Hinblick auf die Symptomatik empfehlenswert, sondern verbessert auch die Prognose, da die bei Nichteinhaltung der Diät persistierende chronische Entzündung das Risiko für ein malignes Lymphom im Dünndarm deutlich erhöht. $\mathrm{Zu}$ den neuen in Entwicklung befindlichen Therapiestrategien gehören das topische Steroid Budesonid, Antikörper gegen Zelloberflächen-Chemokinrezeptoren, Integrine und Polymere, die Gluten selektiv binden.

\section{Wenn die Therapie nicht anschlägt ...} Bei einem therapierefraktären Verlauf sollte zunächst immer an Diätfehler gedacht werden, zumal auch kleinste Mengen an Gluten zu einer Mukosaschädigung führen können. Bei einer echten Therapierefraktärität sollte die Diagnose kritisch überprüft werden, da viele andere Erkrankungen wie M. Crohn, Darmtuberkulose, eosinophile Enteritis, HIV-Infektion oder eine Amyloidose mit einem Zöliakie-ähnlichen Schleimhautumbau einhergehen können. Aber es gibt auch eine echte therapierefraktäre Zöliakie, bei der bestimmte monoklonale autonome Lymphozyten beteiligt sind, die eine hohe Entartungstendenz zeigen, sodass diese Patienten intensiv überwacht werden müssen. 